\title{
Uniform modeling of bacterial colony patterns with varying nutrient and substrate
}

\author{
Deborah Schwarcz ${ }^{1}$, Herbert Levine ${ }^{2,3}$, Eshel Ben-Jacob ${ }^{1,2}$, Gil Ariel $^{4}$ \\ KEYWORDS: pattern formation, bacterial colonies, reaction-diffusion model, random \\ lattice
}

Corresponding author: Gil Ariel, arielg@math.biu.ac.il

\begin{abstract}
Bacteria develop complex patterns depending on growth condition. For example, Bacillus subtilis exhibit five different patterns depending on substrate hardness and nutrient concentration. We present a unified integro-differential model that reproduces the entire experimentally observed morphology diagram at varying nutrient concentrations and substrate hardness. The model allows a comprehensive and quantitative comparison between experimental and numerical variables and parameters, such as colony growth rate, nutrient concentration and diffusion constants. As a result, the role of the different physical mechanisms underlying and regulating the growth of the colony can be evaluated.
\end{abstract}

\section{Introduction}

In nature, bacteria cope with a wide range of environmental conditions. To take advantage of their surroundings, bacteria need to adapt to the external environment using various ways such as variation of movement, communication techniques and adaptation of their rate of reproduction [1-6, 9-11, 14-17, 19-28, 33-34]. If nutrients are deficient for a sufficiently long period of time, some strains of bacteria such as B. subtilis (Bacillus subtilis) enter a special stationary state of a spore, which enables them to survive until more favorable conditions return.

\footnotetext{
${ }^{1}$ School of Physics and Astronomy, Raymond and Beverly Sackler, Faculty of Exact Sciences,

Tel-Aviv University, Tel-Aviv 69978, Israel.

${ }^{2}$ Center for Theoretical Biological Physics, Rice University, Houston, TX 77005, USA.

${ }^{3}$ Department of Bioengineering. Rice University, Houston, TX 77005, USA.

${ }^{4}$ Department of Mathematics, Bar Ilan University, Ramat Gan 5290002, Israel.
} 
Laboratory experiments can test the growth of bacterial colonies under various environmental conditions, for example, by changing the amount of food (the peptone level), or the hardness of the substrate (the agar concentration) on which the bacteria grow in a Petri dish. With plenty of nutrients and a soft substrate, bacteria move individually inside the agar and reproduce at a maximal rate. However, when the agar is hard, bacteria have to cooperate and can only move collectively on top of the substrate.

Environmental conditions change the microscopic behavior of bacteria. As a result, the pattern of the entire colony may change, creating a morphology diagram $[33,17,2]$. Figure 1 shows such a morphology diagram obtained by Rafols [2] for B. subtilis. The diagram is divided into five regions corresponding to different types of patterns, depending on the hardness of the substrate (horizontal axis) and the initial nutrient level (vertical axis) $[2,17]$. For high nutrient concentration and soft substrate, colonies form disk like patterns. However, under harsher conditions, the colony exhibit a fractal-like shape as described in next section, see also [1,5-9, 11-12, 17,18,21, 33, 34]. The main purpose of this article is to present a unified model that reproduces all the different patterns observed in Figure 1 by varying only the initial nutrients level and two parameters that correspond to the hardness of the substrate. This approach allows a comprehensive comparison between experimental and numerical variables and parameters, such as colony growth rate, nutrient concentration and diffusion constants.

Biological background. As explained above, the experimental morphology diagram of $B$ subtilis reproduced from [2,17] in Figure 1 is divided into five regions, each corresponding to a different pattern. With high agar and poor nutrients (region A), the colony has a branched structure. Adding nutrients (region B), branches become thicker and the colony has a round shape. On the other hand, decreasing the agar percentage, and keeping nutrient low (region E), conditions are harsh and a dense branching morphology is observed. However, with sufficiently high nutrients and soft substrate (region D), conditions are favorable and the colony forms an almost full disk. An interesting intermediate region occurs with high nutrient levels but moderate agar concentrations (region C). Then, bacterial colonies of some species, for example B. subtilis, can develop a ring-like pattern, suggesting that the colony alternates between two states - a fast expansion state similar to region D and a slow growth state, similar to region B [18]. Using our simulation results, in the following we give conditions under which ring patterns can occur and explain the phenomenon by alternating modes of movement, represented in the model by different types of diffusion. 
Other bacterial species, for example Proteus mirabilis, have been found to form rings patterns $[18,28,29]$. It has been suggested that rings in Proteus mirabilis colonies are caused by a mechanism of alternating motility phases, more specifically, cyclic process of differentiation and de-differentiation of swimmers into swarmers [2829].

Microscopic observations show that bacterial movement depends on the hardness of the substrate [2-5]. At very low agar concentrations (below 0.3 percent) bacteria move individually inside the agar. However, as agar powder creates a gel, the rigidity of the gel increases with the agar percentage [2-4]. Between 0.3 and 1 percent agar concentration some bacteria move on the surface while some inside [2]. However, bacteria can significantly change the physical properties of the medium, making it more suitable for bacterial movement, by secreting various materials such as enzymes, surfactants and other polymers [1,2]. At higher agar concentrations, the surface is semi-solid and bacteria cannot move at all inside. Instead, bacteria produce a layer of liquid to move in, essentially creating a lubrication layer to increase motility [2,4]. This is a collective endeavor that requires sufficient bacterial density. As a result, at medium and high agar concentrations the ability of bacteria to move depends on its density.

Modeling bacterial colonies. Different theoretical models describing the physical properties underlying pattern formation in bacterial colonies have been suggested and tested by simulations. The general approach underlying the models varies significantly. In [3,63738], emphasis is given on the collective effect of bacteria on their environment. The main goal of [39] is to explore the ability of bacteria to differentiate into different types. Other models aim to expose the possible cell to cell interaction [36]. Many of these and other models take into consideration the influence chemotaxis $[1,523,38]$.

From the point of view of modelling, models can be divided into two main categories.

- Bacteria as discrete agents: The approach models bacteria, or an effective group of bacteria, as discrete self-propelled particles, moving and interacting with each other and the environment. In typical models, agents consume nutrients, multiply, sporulate or die $[5,6,19]$. This approach is particularly advantageous for modeling the internal state of bacteria, for example, energy levels or stress.

- Bacteria as a continuous density: The approach describes the evolution of the bacterial concentration function, typically in two-dimensions (2D) as a partial integro-differential equation, for example a reaction-diffusion equation. Additional constituents of the system, for example, nutrients, signaling factors and other chemicals or proteins are also modelled as a $2 \mathrm{D}$ density. See, for example $[1,7,8,15,34]$. 
In this paper, we follow the second, continuous, approach which allows us to model the large number of bacteria and directly compare simulation and experimental results.

Based on biological observations, we have constructed a unified reaction-diffusion model that reflects the experimental conditions. To our knowledge, this is the first model that explicitly takes into consideration the different component of the substrate hardness; agar concentration and lubricant creation by bacteria. This new description of substrate properties and its influence on the bacterial motion enables us to effectively capture the colony's expansion under different bacterial modes of motion (swarming, swimming). However, the model is essentially a phenomenological reaction-diffusion model. Such a modelling approach neglects many aspects of the bacterial dynamics such as surfactant production, run-and-tumble dynamics, signaling agents and more, which are only captured in a phenomenological, coarse grained nature. None the less, this approach allows a quantitative comparison between experimental and simulation parameters such as diffusion coefficients and the bacterial and nutrient density, see Table 1. The comparison reinforces the applicability of the model in explaining the experimental data. Moreover, the model reproduces all five colony patterns of Figure 1 and enables to relate the different patterns to different types of movement.

Reaction-diffusion models. Typical continuous models for bacteria pattern formation include two or three scalar fields, describing active bacteria density, denoted $b$, nutrient density, $n$, and possibly the density of non-active bacteria or spores, $s$. The rate of change in the bacterial density is generally given by

$$
\frac{\partial b}{\partial t}=\nabla\left(D_{b}(n, b) \nabla b\right)+\beta(n, b) b-\mu(n, b) b .
$$

The first terms describes bacterial movement, the second reproduction and the last mortality. Nutrients diffuse and are being consumed by bacteria,

$$
\frac{\partial n}{\partial t}=D_{n} \nabla^{2} n-3 \beta(b, n) b
$$

The factor 3 multiplying $\beta$ is motivated in [1]. For short, we assume that bacteria need three time its weight in order to reproduce, one quanta for doubling body mass, one quanta used for movement and all other metabolic processes during the reproduction time. 
In our model, inactive (dead or sporulated) bacteria do not influence active ones (active bacteria are located at the extremity of the colony while the inactive ones are inside). The concentration of inactive bacteria merely serves to keep track of the colony's growth history [1]. Accordingly, its rate of change is given by

$$
\frac{\partial s}{\partial t}=\mu(b, n) b
$$

Biological consideration for $D_{b}, \beta$ and $\mu$. It is well known that some bacteria including B. subtilis are able to produce an extracellular "wetting" fluid that enables them to move on top of hard surfaces. Golding et al. [9, 20] model this wetting fluid (also called a lubrication layer) by adding an equation to describe its local height above the agar surface. See appendix A for details. Kitsunezaki [10], includes this dependence by assuming that the diffusion coefficient of the bacteria depends on the bacterial concentration, i.e., $D_{b}=D_{0} b^{k}$. As a result, the movement of bacteria is given by nonlinear diffusion that allows a sharp interface at the edge of the colony, in accordance with experimental observations. In Kawasaki et al. [11], $D_{b}$ depends also on the local nutrient concentration. The Kawasaki model does not include bacterial mortality. Instead, starved bacteria become immobile. Mimura [12] suggested using different diffusion terms for soft and hard agar concentrations. With a hard agar, bacteria move inside the lubrication layer and the diffusion parameter depends on bacterial density. However, when the agar is soft, the bacterial diffusion parameter is constant. Lacasta et al. [39] assume that $D_{b}$ oscillates between different kinds of bacterial diffusion during one simulation.

- Motivated by these approaches, we distinguish between the hard and soft agar cases and define the bacterial diffusion to be $D_{b}=D_{0} b^{k}$ where, the diffusion physical prefactor $D_{0}(a)$ is an decreasing function of agar concentration, as shown in table 1 , where $a$ reflects the substrate hardness. In simulations, $D_{0}$ is determined in each simulation to adjust the experimental time.

- The constant $k$ determines the diffusion type - linear or non-linear. When the agar is soft enough, bacteria swim individually inside the agar and diffusion is independent of the bacterial density, i.e., $k=0$. However, when agar is hard, diffusion increases with the bacterial density, $k=1$.

More precisely, we take $k$ to be a time-dependent parameter satisfying 


$$
k(t+\Delta t)= \begin{cases}0 & a-\delta b \leq 0.6 \\ 1 & a-\delta b \geq 0.63 \\ k(t) & \text { otherwise }\end{cases}
$$

where $a-\delta b$ reflects the substrate hardness, it depends on two parameters: $a$, the percentage of agar $(\mathrm{gr} / 100 \mathrm{ml})$ used in experiments and $\delta\left(10 \cdot \mathrm{cm}^{-1}\right)$, a parameter modeling the effect of bacteria on the substrate through lubricant production (assumed linear for simplicity), we take $\delta=0.077$. When substrate hardness is lower than a given threshold, bacterial diffusion is linear, as in pattern D and E. However, if the substrate hardness is higher than an upper limit, bacteria move collectively on the substrate and the lubricating liquid has a local influence on the bacterial movement, as in pattern A and B. This implies that the bacterial diffusion should depend on the bacterial density, i.e., $k>0$ , indicating a non-linear diffusion. See appendix A for details. We assume a coexistence state between the two thresholds - if substrate hardness is below the upper limit but did not yet reach the lower one, bacterial diffusion will stay non-linear because hardness substrate is not sufficiently small to require bacterial re-adaptation to the substrate hardness. In the same way, if hardness substrate increases above the lower threshold but did not yet reach the upper one, bacterial diffusion will stay linear. The gap between the threshold values for linear and non-linear diffusions causes a mixed coexistence state in which both motion types can occur. The gap is crucial for formation of a robust ring pattern. In particular, rapid switching between the two diffusion types at an unrealistically high frequency may blur the ring pattern or smooth it out.

The model also includes an adaptation of the bacterial reproduction rate, $\beta$, to the average nutrient concentration, $N$. In order to be as close as possible to experimental findings, the reproduction rate, $\beta$, is assumed to depend on a global parameter, reflecting the relation between the composition of the substrate and the bacterial growth rate. When nutrients are abundant, the reproduction rate is taken to be constant. However, when the nutrients are low, we assume that bacteria reproduction depends linearly on local nutrients amount, as follows;

$$
\beta= \begin{cases}0.3 & N>0.9 \\ n & N \leq 0.9\end{cases}
$$

where $N$ is the nutrients average given by, 


$$
N=\frac{\int_{B} n d x d y}{\int_{B} d x d y} .
$$

Here, $B$ is defined as,

$$
B=\left\{(x, y) \in A \mid b(x, y)>b_{\min }\right\} \text { with } b_{\min }=0.05 b_{\max } .
$$

In other words, bacteria in the colony can be in one of two states: in the first, reproduction rate is constant, while in the second it depends on the local nutrient concentration. Experiments show that the reproduction process includes DNA replication beginning when the cell mass per origin chromosome reaches a particular value [32]. Once initiated, DNA replicates at a constant rate [16]. In addition, during the solidification phase of the concentric rings pattern $(\mathrm{C})$, the reproduction rate is doubled because the colony invests more resources to reproduce and less in movements and lubricant production.

Note that $N$ is determined by a global condition rather than a local one. This reflects the observation that bacteria in a colony can act in cooperation [22]. In our model, the qualitative form of the reproduction rate depends on a collective property of the entire colony. A possible mechanism that allows global colony-wide communication and coordinated growth rates has been suggested in [27] using a quorum sensing mechanism and involving several chemical communication agents. The physical details of this complex mechanism are beyond the scope of this paper. As a result, in order to simplify our model, we assume a global condition for the reproduction rate in the case of nutrient abundance. More importantly, we observe that replacing the global condition with an analogue local one does not yield satisfactory results, especially in forming uniformly growing ring patterns. This observation confirms the hypothesis suggested in [27] regarding a sophisticated colony-wide communication channels allowing bacteria to coordinate their internal state.

Finally, the mortality rate, $\mu$, is assumed to depend on the local nutrients levels as $\mu=1 /(1+4 n)$. This implies that that without nutrients, bacteria will become inactive (or spores) with rate, $\mu(n=0)=1$, while with a high concentration of nutrients, sporulation stops, $\mu(n>>1)=0$. Note that the physical meaning of dimensionless expressions such as $1+4 n$ can only be interpreted once a scaling between simulation and physical parameters is given, see the results section and Table II. 
Implementation. The model equations described above are solved numerically using a random computational lattice $[10,13,14]$, which artifact is useful to eliminate symmetries due to the underlying lattice. We applied a Vectorizable Random Lattice, as detailed in appendix B.

Initially, the active bacteria, $b$, are distributed in a circle at the middle of the plate with smooth edges given by $b(x, y, t=0)=H\left(0.075 \sqrt{x^{2}+y^{2}}\right)$, where,

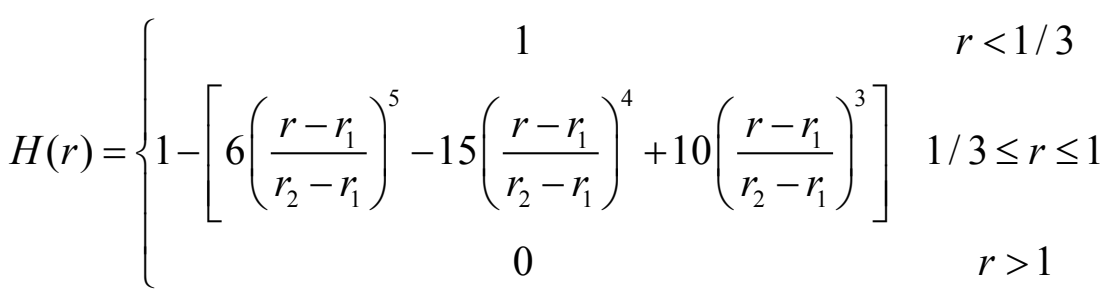

Nutrients have an initial uniform distribution, $n(x, y, t=0)=n_{0}$ and $s(x, y, t=0)=0$.

\section{Results}

Numerical solutions with varying substrate hardness and initial nutrient concentration reproduce all the five bacterial patterns depicted in figure 1. Parameter values are listed in table. I.

We find that the colony's expansion rate, depicted in Figure 2, is approximately linear in patterns A, B, D, E. This is in accordance with experimental measurements [2].

Of particular interest is the periodic ring-like pattern $\mathrm{C}$. When agar-concentration is at a medium level ( $a=0.7$ ), the bacterial movement alternates between linear and non-linear diffusion motions within a single simulation. The reason for this periodic behavior, as observed in simulations (see figure 4), is as follows: When the bacterial density reaches an upper limit they can create sufficient fluids, and the substrate become soft, enabling rapid motion due to the linear diffusion. As a result of the high expansion rate, the bacterial density at the edge of the colony decreases, increasing substrate hardness at the edge until the lower threshold is reached. This implies that bacteria cannot create enough fluid and therefore have a reduced motility through non-linear diffusion. The average bacterial density at the edge pattern $\mathrm{C}$ is show in figure 3 . 
This behavior explains the ring like pattern in the experimentally observed pattern $\mathrm{C}$ (figure 1). The growth of the colony is divided into two behavioral characteristics: consolidating and migrating. It has been found that a concentric ring-like pattern is formed by alternating the region D-like colony expansion (very fast) and the region Blike growth (very slow) [18]. These two phases occur one after the other periodically, each step lasting a few hours. Figure 4 show these two phases. When the substrate is soft bacteria move using the fluidity of the substrate and the diffusion is linear.

This is the first model that takes into consideration the different component of the substrate hardness; agar concentration and lubricant creation by bacteria. This new description of substrate softness and its influence on the bacterial motion enables us to reproduce the variety of bacterial motion. Diversity of bacterial motion reflects bacterial ability to adapt themselves to different environmental conditions [2, 20, 21]. Moreover, the model enables a quantitative comparison between experimental and simulation parameters, reinforcing its applicability to the experimental data.

Quantitative comparison between experiments and simulations. The purpose of this section is to show a direct correspondence between the physical quantities used in experiments and numerical values in simulations. We begin by comparing temporal, spatial and mass scales. Length scale: Both simulations and experiments terminate when a colony is about half-way through to the edge of the petri dish (diameter $8.8 \mathrm{~cm}$ ) or the simulated domain 60 cells, on a lattice of $120 * 120$ cells. Accordingly, each numerical cell is equivalent to roughly $0.7 \mathrm{~mm}$.

In experiments, growth to a diameter of about $5 \mathrm{~cm}$ required about a month in region $\mathrm{A}, \mathrm{a}$ week in region $\mathrm{B}$, a day in regions $\mathrm{C}$ and $\mathrm{E}$, and half a day in region $\mathrm{D}$. In simulations, patterns $\mathrm{A}, \mathrm{B}, \mathrm{D}$ and $\mathrm{E}$ exhibit a constant growth rate as shown in figure 2. Growth rate of pattern $\mathrm{C}$ has two phases, a consolidation phase in which colony radius stay constant and a migrating phase in which the colony expand as show in figure 4.

Figure 5A shows that all growth rates are linearly dependent, indicating that each time step in the numerical simulation is equivalent to about one hour. We note that the comparison between simulated and experimental times should only be understood as a mean to estimate the scaling and orders of magnitude between model parameters and physical constants. For example, the initial conditions in simulations and experiments are fundamentally different. Experiments report a lag time until the colony starts to expand. This lag time, and possibly also the initial distribution of bacteria, depends on the experimental conditions. This is just another detail that is not been considered in our model. 
Figure 4B shows the experimental initial nutrients concentration vs. the analogue parameter, $n_{0}$, used in simulating the different patterns. This establishes the relation between the physical and numerical mass units, $b$ and $s$ are measured in number of bacteria per $\mathrm{cm}^{2}$. Hence, we can convert it to units of $\mathrm{mg}$ per area by multiplying the bacterial number by $9.5 \cdot 10^{-13} \mathrm{~g}$, the average weight of one bacteria.

The nutrient field, $n$, is either measured in $\mathrm{gr} / \mathrm{cm}^{2}$ or $\mathrm{gr} / \mathrm{liter}$. Using the considerations described above, $1 \mathrm{gr} / 1$ corresponds to $0.3 \mathrm{mg} / \mathrm{cm}^{2}$. There is a linear correlation between the nutrient amount during the experiments and the initial nutrient amount of the simulation. Table II details the correspondence between all experimental simulation values.

\section{Discussion}

In this work we develop a reaction-diffusion model that takes into account both the physical properties of the substrate and the way bacteria can modify it. Both mechanisms affect the properties of the bacterial motion. This allows us to reproduce all five patterns appearing in the morphology diagram of $B$. Subtilis within a single unified model. In region A, the non-linear diffusion creates branched patterns. Increasing the initial nutrient concentration, the gap between branches decreases, producing a thicker pattern, labelled $B$. These two cases have the same type of diffusion (non-linear) and also the same diffusion coefficient. The colony's expansion rate is lower for Pattern A than in B since the initial nutrient concentration in A is lower. As a result, simulations show that bacteria become in-active at a higher rate. Because in this regime, diffusion is non-linear, smaller $b$ implies effectively reduced motility. In the case of nutrient abundance and soft agar we get a disk like pattern colony, as in region D. In this case, diffusion is linear and reproduction rate is constant. Reducing the initial nutrients, the diffusion stays linear. However, the reproduction rate, which in this regime depends on the local amount of nutrients, decreases. This dependence of the reproduction rate on location is the source of the irregular fractal-like pattern E. Finally, when nutrients are high and agar concentration is at an intermediate value, the mobility of bacteria alternates between linear and non-linear diffusion. As described in the results section, when the density is sufficiently high, it has a critical influence on the substrate; by emitting fluid bacteria change the substrate from a medium-hard substrate on which they hardly move (nonlinear diffusion) to a soft substrate on which they move easily (linear diffusion). This depends on the colony capacity to reach a critical density. 
The unified modelling approach allows a comprehensive comparison between the experimental and simulated parameters and values. This is particularly important in complex models involving a large number of fitted parameters. In particular, we found linear relations between the colony's growth rates and the initial nutrient concentration used in simulation and the experimental values across all different patterns. Diffusion coefficients and bacterial densities are also in accordance with experimental measurements.

\section{Acknowledgments}

We thank Ismael Rafols for sharing experimental results and electronic copies of figures 1A and 4A. This article has been supported in part by the NSF Center for Theoretical Biological Physics (Grants No. PHY-1427654 and No. NSF-MCB-1214457) at Rice University and by the Tauber Family Funds and the Maguy-Glass Chair in Physics of Complex Systems at Tel Aviv University 


\section{References}

1. Ben-Jacob E, Cohen I and Levine H (2000) Cooperative self-organization of microorganisms, advances in physics 49: 395- 554.

2. Rafols I, Formation of Concentric Rings in Bacterial Colonies (1998) MSc thesis, Chuo University, Japan.

3. Cohen I, Golding I, Ron G, Ben-Jacob E (2001) Biofuid dynamics of lubricating bacteria, Mathematical Methods in the Applied Sciences, 24:1429-1468.

4. Kearns DB (2010) A field guide to bacterial swarming motility, nature reviews. Microbiology, 8: 634-644.

5. Ben-Jacob E, Sochet O, Tenenbaum A, Cohen I., Czirok A et al. (1994) Generic Modeling for Cooperative Growth Patterns of Bacterial Colonies, Nature 368: 4649.

6. Cohen I, Golding I, Kozlovsky Y, Ben-jacob E (1999) Continuous and discrete models of cooperation in complex bacterial colonies, Fractals 7: 235-247.

7. Mimura M, Sakaguchi H, Matsushita M (2000) Reaction-Diffusion modeling of bacterial colony patterns, Physica A, 282: 283-303.

8. Matsushita M, Wakita J, Itoh H, Watanabe K, Arai T et al (1999) Formation of colony patterns by a bacterial cell population, Physica A 274: 190-199.

9. Golding I, Kozlovsky Y, Cohen I, Ben-Jacob E (1998) Studies of Bacterial Branching Growth using Reaction-Diffusion Models for Colonial Development, Physica A 260: 510-554.

10. Kitsunezaki S, (1999) Interface Dynamics for Bacterial Colony Formation Journal of Physical Society of Japan 66: 1544-1550

11. Kawasaki K, Mochizuki A, Matsushita M, Umeda T, Shigesada N (1997) Modeling Spatio-Temporal Patterns Generated by Bacillus Subtilis, Journal of Theoretical Biology 188: 177-185.

12. Mimura M, Sakaguchi H, Matsushita M (2000) Reaction-diffusion modeling of bacterial colony patterns, Physica A 282: 283-303.

13. Moukarzel C, Herrmann H.J (1992) A Vectorizable Random Lattice, Journal of Statistical Physics 68: 911-923.

14. Tucker L.J (2010) A new computational approach to simulate pattern formation in Paenibacillus dendritiformis bacterial colonies, Ph.D. thesis, UC San Diego: b6691544. Available: https://escholarship.org/uc/item/6x91c78r.

15. Be'er A, Smith S.R, Zhang H.P, Florin E.L, Payne S. N et al. (2009) Paenibacillus dendritiformis Bacterial Colony Growth Depends on Surfactant but Not on Bacterial Motion, Jornal of Bacteriology, 191(18): 5758-5764.

16. Scheffers, D.J (2013) Bacterial Reproduction and Growth. In: eLS. John Wiley \& Sons Ltd, Chichester. 
17. Matsushita M, Hiramatsu F, Kobayashi N, Ozawa T, Amazaki Y et al. (2005) Colony formation in bacteria: experiments and modeling, Biofilms, 1: 305-317.

18. Shimada H, Ikeda T, Wakita J, Itoh H, Kurosu S et al. (2003), Dependence of Local Cell Density on Concentric Ring Colony Formation by Bacterial Species Bacillus subtilis, Journal of the Physical Society of Japan, 73: 1082-1089.

19. Czirok, A., Ben-Jacob, E., Cohen, I. and Vicsek T. (1996), Formation of complex bacterial colonies via self-generated vortices, Physics review Letters E, 54: 17911801

20. Kozlovsky Y, Cohen I, Golding I, and Ben-Jacob E (1999) Lubricating bacteria model for branching growth of bacterial colonies, Physics review Letters E, 59: 7025-7035

21. Kinsinger R.F, Shirk M.C, and Fall R. (2003) ) Rapid surface motility in Bacillus subtilis is dependent on extracellular surfactin and potassium ion, Journal of bacteriology, 185: 5627-5631

22. Niu B. and Wang H (2012), Bacterial Colony Optimization, Discrete Dynamics in Nature and Society.

23. Baronas R. and Simkus R, (2012), Modeling and Simulation of Bacterial SelfOrganization in Circular Container Along Contact Line as Detected by Bioluminescence Imaging, International Journal on Advances in Systems and Measurements. 5:154-163

24. Tyson R, Stern L.G and LeVeque R.J (2000), Fractional step methods applied to a chemotaxis model, Journal of mathematical biology, 41: 455-475

25. Tyson R, Lubkin S.R, Murray J.D (1999), A minimal mechanism for bacterial pattern formation, The royal society, 266 299-304..

26. Christopher M, Waters and Bonnie L. Bassler (2005), Quorum Sensing: Cell-toCell Communication in Bacteria, Annual Review of Cell and Developmental Biology., 21:319-46.

27. Ben Jacob E, Lu M, Schultz D and Onuchic J.N (2014), The Physics of Bacterial Decision Making, Frontiers in Cellular and Infection Microbiology, 4:154.

28. Xue, C, Budrene E.O, and Othmer H. G. (2011). Radial and spiral stream formation in Proteus mirabilis colonies, PLoS comput. biology

29. Matsuyama T, Takagi Y, Nakagawa Y, Itoh H, Wakita J, et al. (2000) Dynamic aspects of the structured cell population in a swarming colony of Proteus mirabilis. Journal of Bacteriology 182: 385-393.

30. Lega J (2011) Collective Behaviors in Two-Dimensional Systems of Interacting Particles, Journal on applied dynamical system, 10:1213-1231

31. Maury B, Roudneff-Chupin A and Santambrogio F (2014) Congestion driven dendritic growth, Discrete and Continuous Dynamical Systems, 34: 1575-1604

32. Suckjoon J. and Sattar T.A (2015), Cell-Size maintenance: universal strategy revealed, Trends in Microbiology, 23:4-6 
33. Ohgiwari M, Matsushita M and Matsuyama T (1991), Morphological Changes in Growth Phenomena of Bacterial Colony Patterns, Physical Society of Japan, 61:816-822.

34. Matsushita M, Wakita J, Itoh H, Rafols I, Matsuyama T et al. (1998), Interface growth and pattern formation in bacterial colonies, Physica A 249:517-524.

35. Wu Y, Kaiser D, Jiang Y, Alber M (2008), Periodic reversal of direction allows Myxobacteria to swarm, Proceedings of the national academy of sciences of the U.S.A, 106:1222-1227.

36. Wu Y, Jiang Y, Kaiser D, Alber M (2007), Social interactions in myxobacterial swarming, PLoS Computational Biology 3 e253.

37. Du H, Xu Z, Anyan M, Kim O, Leevy W.M and Shrout J.D. (2012), High Density Waves of the Bacterium Pseudomonas aeruginosain Propagating Swarms Result in Efficient Colonization of Surfaces, Biophysical Journal 103:601-609.

38. Bees, M. A, P. Andresen, Mosekilde E, M. Givskov (2002) Quantitative effects of medium hardness and nutrient availability on the swarming motility of Serratia liquefaciens, Bulletin of Mathematical Biology 64:565-587.

39. Lacasta A.M, Cantalapiedra I.R, Auguet C.E, Penaranda A. and L. RamirezPiscina (1999), Modelling of spatio-temporal patterns in bacterial colonies, Physical Review E 59:7036.

40. Angelini T.E., Roper M., Kolter R., Weitz D.A. and Brenner M.P., Bacillus subtilis spreads by surfing on waves of surfactant (2009), Proc. Nat. Acad. Sci. USA 106, 18109-18113. 


\section{Appendix A: bacterial lubricant effect on bacterial movement}

Recall equation (4) describing the type of bacterial diffusion: linear for soft agar or nonlinear when bacteria move collectively within a self-generated lubricating fluid. In the second case, the bacterial diffusion depends on the height of the lubricating fluid. Under certain assumptions, one can describe the dependence of the bacterial diffusion on the bacterial density.

In order to derive this, we will present a model taking into consideration the lubricating fluid, secreted by the active bacteria and its influence on the active bacterial motion. Then, we present some assumptions leading to a description of the fluid influence on the active bacterial motion through the active bacterial density [1].

Golding et. al. [6] suggest an equation that describes the rate of change in the lubricant fluid height, $l$ as:

$$
\frac{\partial l}{\partial t}=-\nabla \vec{J}_{l}+\Gamma b n\left(l_{\max }-l\right)-\lambda l
$$

where $\vec{J}_{l}$ is the fluid flux, $\Gamma$ is the rate of the fluid production and $\lambda$ is the absorption rate of the fluid by the agar. In this model, fluid production depends on three parameters: active bacterial density, nutrients density, and height of the lubrication fluid. The production rate decreases as the amount of the lubrication fluid increase. However, the production term cannot become negative because the lubrication height cannot exceed a maximum threshold $l_{\max }$. In addition, the model assumes that the absorption of fluid into the agar depends on the local fluid height. Because each bacterium drags itself along the fluid surrounding it, the motion of the lubrication fluid depends on the flux of active bacteria. Since the fluid also diffuses, the fluid flux is described as:

$$
\overrightarrow{J_{l}}=-D_{l} l^{\eta} \nabla l+j \overrightarrow{J_{b}}
$$

where $D_{l}$ is the lubrication diffusion coefficient, $\overrightarrow{J_{b}}$ is the active bacterial flux and $j$ is the amount of fluid dragged by each bacterium. The diffusion term of the fluid depends on the height of the fluid to the power of $\eta$. Non-linearity causes the fluid to have a sharp boundary at its front, as observed in the experiments of the development of bacterial colonies.

In order to describe the active bacterial flux $\overrightarrow{J_{b}}$, the model assumes that the friction is inversely related to the local lubrication height through some power law: friction $\propto l^{\gamma}$ 
with $\gamma<0$. As the bacterial motion is over-damped, the local speed of the bacteria (also the mean step length, neglecting collisions between bacteria) is proportional to the selfgenerated propulsion force divided by the friction. It can be shown that variation of the step length leads to variation of the diffusion coefficient, with the diffusion coefficient proportional to the step length to the power of two [1]. As a result, the bacterial flux is

$$
\overrightarrow{J_{b}}=-D_{b} l^{-2 \gamma} \nabla b .
$$

Substituting (A1) and (A2) into (A3) yields,

$$
\frac{\partial l}{\partial t}=\nabla\left(D_{l} l^{\eta} \nabla l+j D_{b} l^{-2 \gamma} \nabla b\right)+\Gamma b n\left(l_{\max }-l\right)-\lambda l
$$

The equation describing the rate of change of the bacterial density is then,

$$
\frac{\partial b}{\partial t}=D_{b} \nabla\left(l^{-2 \gamma} \nabla b\right)+b n-\mu b .
$$

Active bacteria diffuse (first term), reproduce (second term) and become inactive (last term). As noted above, the diffusion of the active bacterial density depends on the lubrication height. In this case, the flux of the active bacterial density depends on time and position. In $[6,9]$, such a model was used to reproduce the branching pattern A.

The following assumptions enable to describe the influence of the fluid by the dependence of the active bacterial density on the bacterial diffusion [1]:

- The production of lubricant, is proportional to the active bacterial density by a power of $\alpha>0$ so that $l \propto b^{\alpha}$.

- The lubricant is partially absorbed in the agar; the height of the lubricant that is absorbed in the agar is proportional to the lubricant height, which is also proportional to the lubrication height to the power of $\beta>0$

The production and absorption of lubricant occurs at times scales that are significantly shorter than diffusion scales. As a result, we can assume that the lubricant's height is proportional to the active bacterial density to the power of $\alpha / \beta$.

As noted above, we also assume that the friction is proportional to the lubricant's height to the power of $\gamma<0$. Given the above assumptions, the lubrication field can be removed 
from the dynamics and be replaced by a density dependent diffusion coefficient. This coefficient is proportional to the bacterial density to the power of $k=-2 \gamma \alpha / \beta \geq 0$. To summarize, the diffusion term can be written as $\nabla\left(D_{b} b^{k} \nabla b\right)$. In the case of a hard substrate, when bacteria need to extract a lubricant fluid in order to move (patterns A and $\mathrm{B}$ in figure 1), $k>0$. Simulation results for different $k$ are shown in figure A1.

When the substrate is soft enough, bacteria can move individually which suggests taking $k=0$. As we can see from figure 3 , there is a significant, qualitative difference between $k=0$ and $k>0$ cases. However, the precise value of $k$ will only produce small quantitative differences between the patterns as long as it is strictly positive (and not too large). In general, large values of $k$ typically imply wider, more separated fingers. Therefore, in our model we take either $k=0$ or $k=1$.

\section{Appendix B: Vectorizable random lattices}

In many examples requiring a numerical solution for complicated PDE models, symmetries in the underlying computational lattice can induce similar symmetries in the solution. To overcome this difficulty, we applied a Vectorizable Random Lattice (VRL). In a VRL, the sites are a set of randomly chosen points with uniform distribution. In order to construct the VRL, one defines a square lattice, called the reference lattice. Next, draw a random point in each cell while keeping a minimum distance between points. Any two points that are closer than this minimum distance are re-drawn. The minimum distance between the points controls the degree of randomness of the lattice.

In order to define the lattice cells, we compute the Voronoi diagram: Given a set $N$ of $M$ points, $N=\left\{n_{1}, n_{2}, \ldots, n_{m}\right\}$, the Voronoi diagram is a partition of the domain into regions $V\left(n_{I}\right)$ such that any point in $V\left(n_{I}\right)$ is closer to node $n_{I}$ than to any other point $n_{J} \in N(J \neq I)$. The region $V\left(n_{I}\right)$ is the first-order Voronoi cell for a node $n_{I}$,

$$
V\left(n_{I}\right)=\left\{x \in R^{d}: d\left(x, x_{I}\right)<d\left(x, x_{J}\right) \forall J \neq I\right\},
$$

where $d(\cdot, \cdot)$ is the Euclidean distance and $x_{I}$ is the position of node $n_{I}$. Nodes that have a common Voronoi facet are called natural neighbors. Connecting the natural neighbors creates the Delaunay tessellation, thus defining the random lattice. Distances and angles between natural neighbors are not constant, enabling translational and rotational 
symmetry. Once the lattice connectivity has been found, it is required to approximate the model equations in term of discrete variables defined on the nodes of the lattice.

For example, the Laplacian of a function $\varphi$ at lattice point $i$ is computed as [7]

$$
\left.\left(\nabla^{2} \varphi\right)\right|_{i}=\sum_{j} w_{i j} \cdot\left(\varphi_{j}-\varphi_{i}\right)
$$

Here, $j$ are the natural neighbors sites, $w_{i j}$ are coupling constants that depend on the geometry of the Voronoi cells $i$ and $j$. The weight of the neighbors grows in accordance with the length of the common face that separates them in the Vornoi diagram, $f_{i j}$ and becomes smaller as the distance to the neighbor site, $l_{i j}$, gets larger. Lastly, the constant is normalized by the area of site $i$ 's Voronoi cell, $a_{i}$.

Mathematically, the weight of each natural neighbor $w_{i j}$ is given by

$$
w_{i j}=\frac{f_{i j}}{l_{i j} a_{i}} .
$$

It is important to note that the suggested form for (B2) and (B3) conserve mass. The mass in each unit cell is given by the concentration multiplied by the area. Therefore, with a non-uniform grid, in order to verify conservation of mass, one need to write the evolution equations for $m_{i j}=a_{i j} \varphi_{i j}$. The resulting numerical scheme is similar to (B2) and (B3), only with modified weights $w_{i j} a_{i j}=f_{i j} / l_{i j}$, which symmetric with respect to interchanging $i$ and $j$.

In order to demonstrate the Laplacian definition (B2) of a random lattice, we need to divide one Vornoi cell into triangles. Figure B2 depicts a division of a Vornoi cell into triangles.

As noted above, the link between two lattice sites is perpendicular to the Voronoi facet. Therefore, if the triangle base is the Vornoi facet $f_{i j}$, then the triangle height is $l_{i j} / 2$ (as it was noted before, the Vornoi facet or the continuation of it crosses the link of the Delaunay tessellation in the middle). As a result, the area of one triangle that makes the Vornoi cell can be described by the formula:

$$
a_{i j}=\frac{1}{2} f_{i j}\left(\frac{1}{2} l_{i j}\right)=\frac{1}{4} f_{i j} l_{i j} .
$$


Finally, the area of the Vornoi cell is obtained by summing all the areas of the triangles that construct it,

$$
a_{i}=\sum_{j} \frac{1}{4} f_{i j} l_{i j}
$$

Combining (B3) with (B4) and (B5) yields,

$$
w_{i j}=\sum_{j} \frac{4}{l_{i j}^{2}}
$$

A more formal demonstration of $w_{i j}$ is based on a Taylor series of the function $\phi$ at point $i$ [12]. Expanding to second order in the lattice constant,

$$
\varphi_{j}=\varphi_{i}+\left.\left(x_{j}-x_{i}\right) \cdot \varphi_{x}\right|_{i}+\left.\left(y_{j}-y_{i}\right) \cdot \varphi_{y}\right|_{i}+\frac{1}{2}\left[\left.\left(x_{j}-x_{i}\right)^{2} \cdot \varphi_{x x}\right|_{i}+\left.\left(y_{j}-y_{i}\right)^{2} \cdot \varphi_{y y}\right|_{i}\right]+\left.\left(x_{j}-x_{i}\right)\left(y_{\mathrm{j}}-y_{i}\right) \varphi_{x y}\right|_{i} .
$$

Next, let $\alpha_{i j}$ denote the angle between the line connecting sites $i$ and $j$ and the $\mathrm{x}$ axis (See figure B3).

We have,

$$
\begin{aligned}
& x_{i}-x_{j}=l_{i j} \cos \alpha_{i j} \\
& y_{i}-y_{j}=l_{i j} \sin \alpha_{i j}
\end{aligned}
$$

To get the same expressions as in eqn. (B7) one can add 180 degree to $\alpha_{i j}$, and consider $\theta_{i j}$ instead. In this way we get,

$$
\begin{aligned}
& x_{j}-x_{i}=l_{i j} \cos \theta_{i j} \\
& y_{j}-y_{i}=l_{i j} \sin \theta_{i j}
\end{aligned}
$$

Substituting into eqn. (B7) yields,

$$
\begin{aligned}
& \varphi_{j}-\varphi_{i}= \\
& \left.l_{i j} \cos \theta_{i j} \cdot \varphi_{x}\right|_{i}+\left.\left(l_{i j} \sin \theta_{i j}\right) \cdot \varphi_{y}\right|_{i}+\frac{1}{2}\left[\left.\left(l_{i j} \cos \theta_{i j}\right)^{2} \cdot \varphi_{x x}\right|_{i}+\left.\left(l_{i j} \sin \theta_{i j}\right)^{2} \cdot \varphi_{y y}\right|_{i}\right] \\
& +\left.\left(l_{i j} \cos \theta_{i j}\right)\left(l_{i j} \sin \theta_{i j}\right) \varphi_{x y}\right|_{i} .
\end{aligned}
$$

We simplify eqn. (B10) by averaging angles over $[0,2 \pi]$, 


$$
\varphi_{j}-\varphi_{i}=\frac{1}{4}\left[\left.l_{i j}^{2} \cdot \varphi_{x x}\right|_{i}+\left.l_{i j}^{2} \cdot \varphi_{y y}\right|_{i}\right]=\frac{1}{4} l_{i j}^{2}\left[\left.\varphi_{x x}\right|_{i}+\varphi_{y y} \mid\right]_{i}
$$

Combining (B6) and (B11) we yields,

$$
\sum_{j} w_{i j} \cdot\left(\varphi_{j}-\varphi_{i}\right)=\left.\varphi_{x x}\right|_{i}+\left.\varphi_{y y}\right|_{i}=\left.\left(\nabla^{2} \varphi\right)\right|_{i}
$$

The discretization of $\left.[\nabla \cdot(\psi \nabla \varphi)]\right|_{i}$ is obtained in a similar manner. We find that,

$$
\sum_{j} \frac{1}{2} w_{i j}\left(\psi_{j}+\psi_{i}\right) \cdot\left(\varphi_{j}-\varphi_{i}\right)=\left(\psi \varphi_{x x}+\psi \varphi_{y y}+\psi_{x} \varphi_{x}+\psi_{y} \varphi_{y}\right)=\nabla[\psi(\nabla \varphi)]
$$




\section{Figures}
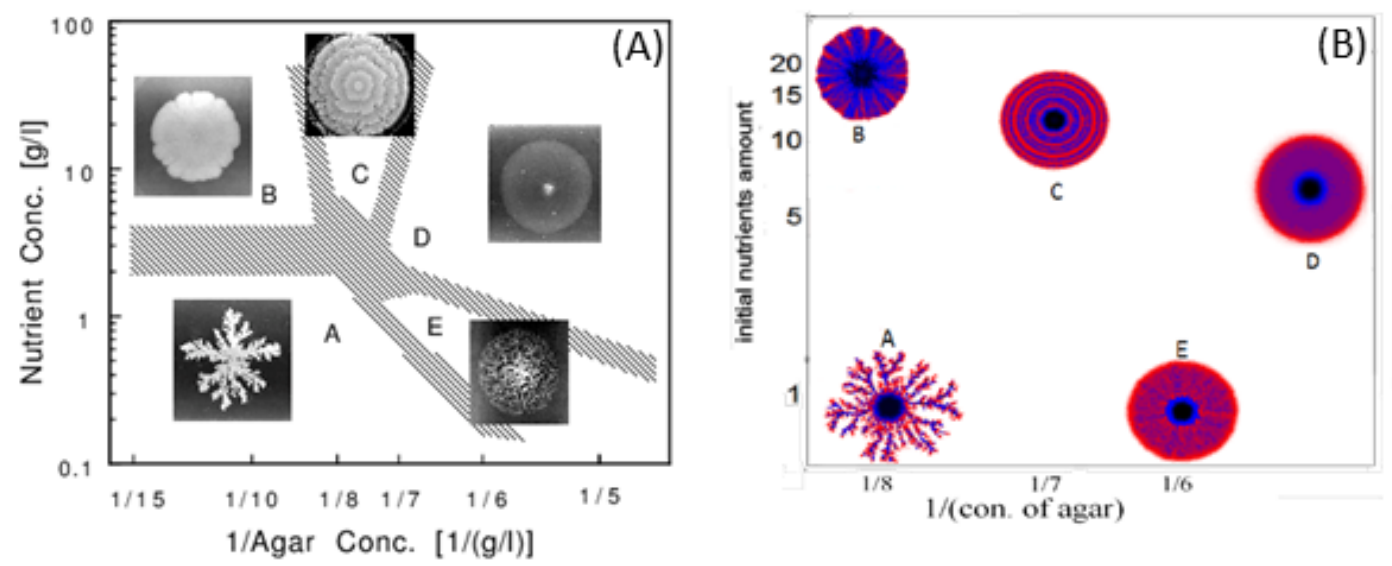

Figure 1 (A) Experimental morphology diagram of B. Subtilis, reproduced from [2]. (B) Simulated patterns reproduce the experimental results. Simulations vary in initial nutrient concentration ( $\mathrm{y}$ axis), agar concentration ( $\mathrm{x}$ axis) and the bacterial diffusion coefficient. 

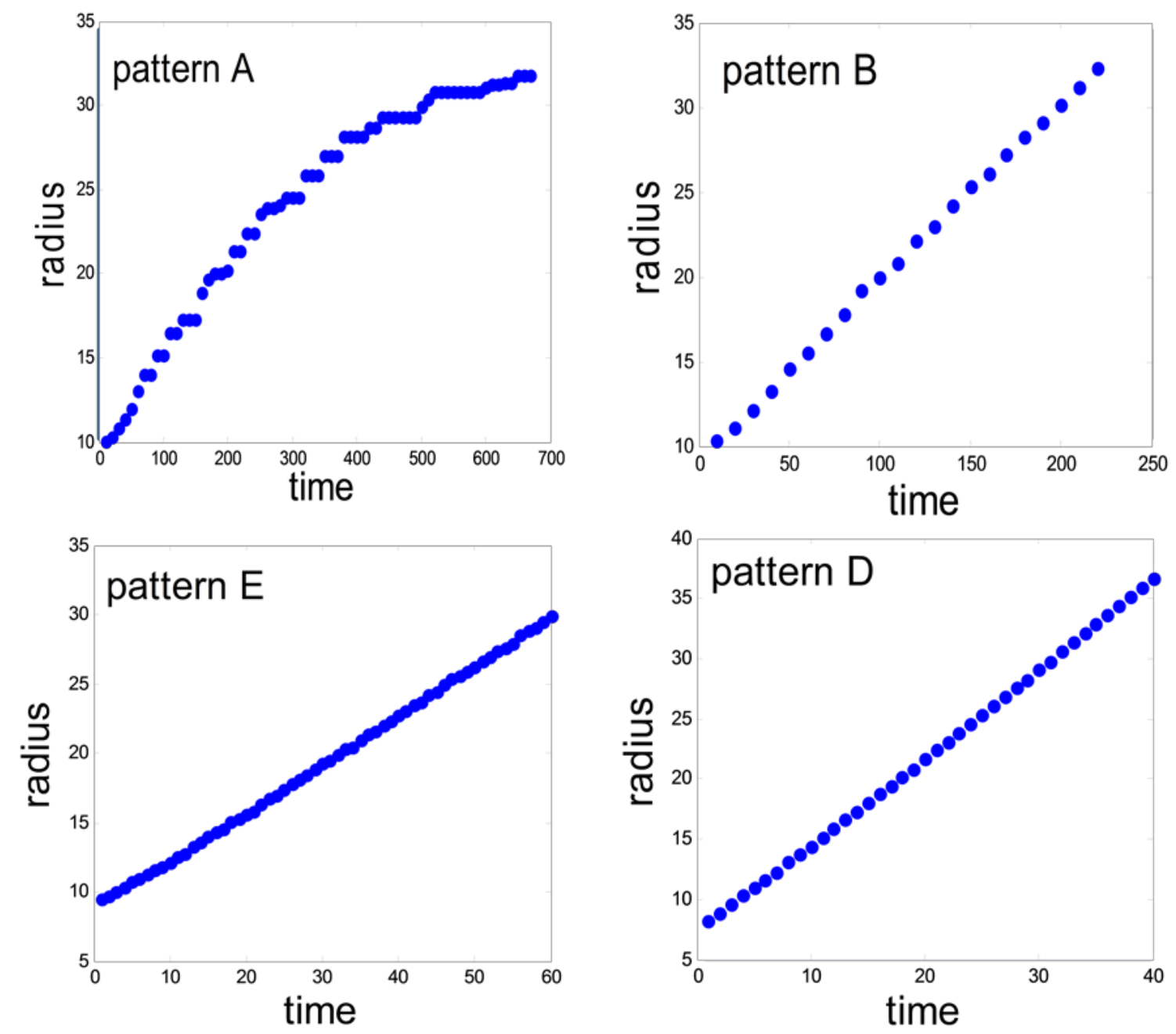

Figure 2 Simulated growth rates. The radius of the simulated colony as a function of time for patterns A, B, D and E. Linear growth is observed. 


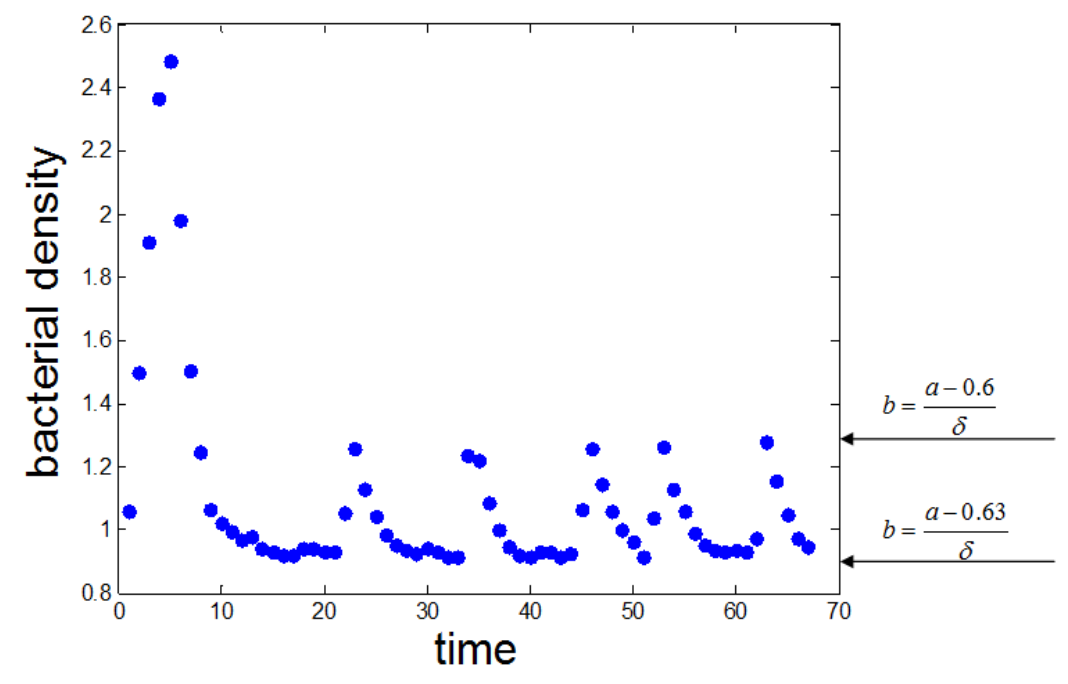

Figure 3 The average bacterial density at the edge pattern $\mathrm{C}$ as a function of time. Arrows show threshold densities at which the type of diffusion changes.
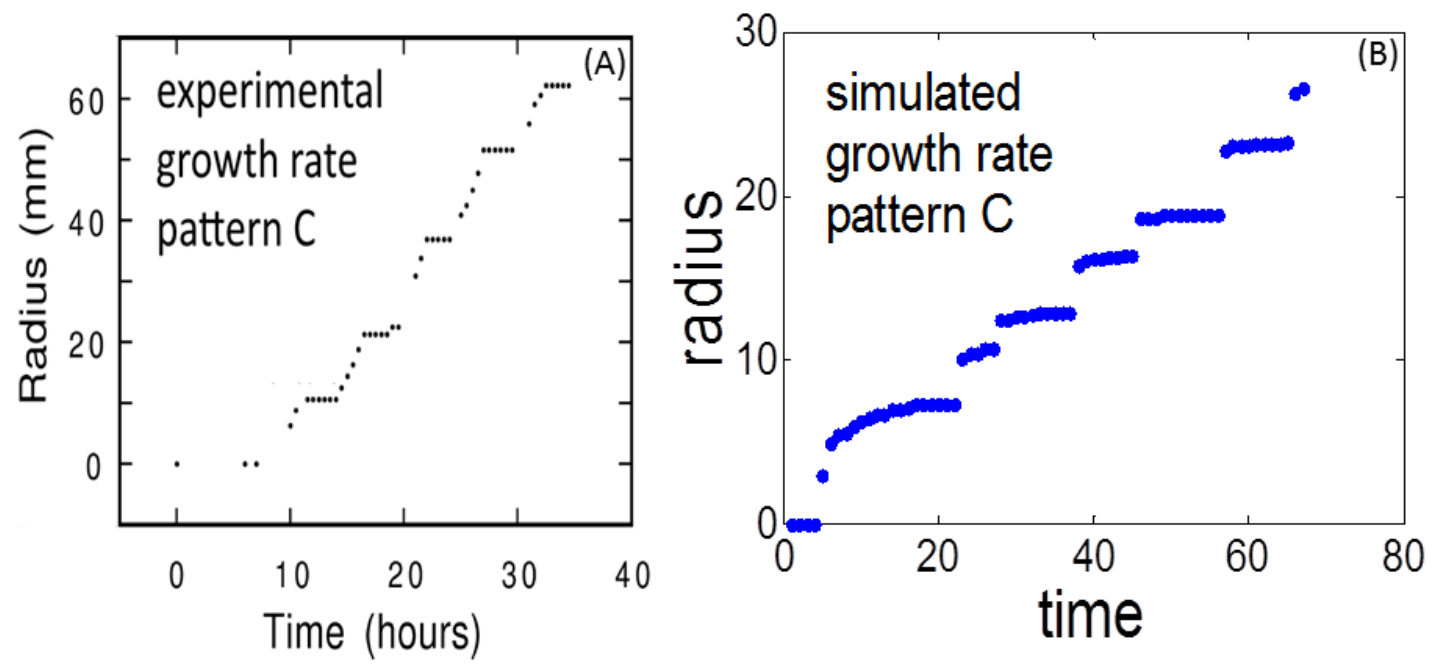

Figure 4 (A) Experimental growth rate of $B$ subtilis ring pattern (pattern C), when agar percentage is $0.76 \mathrm{gr} / \mathrm{l}$. . The radius of the colony pattern as a function of time showing the consolidating and migrating growth rates. Reproduced from Rafols [2]. Notice that in this experiment, the final radius of the pattern is $6 \mathrm{~cm}$. (B) Simulated growth rate of pattern $\mathrm{C}$. The size of the step-like jump decreases as a function of the minimal bacterial density used to define the pattern radius. 

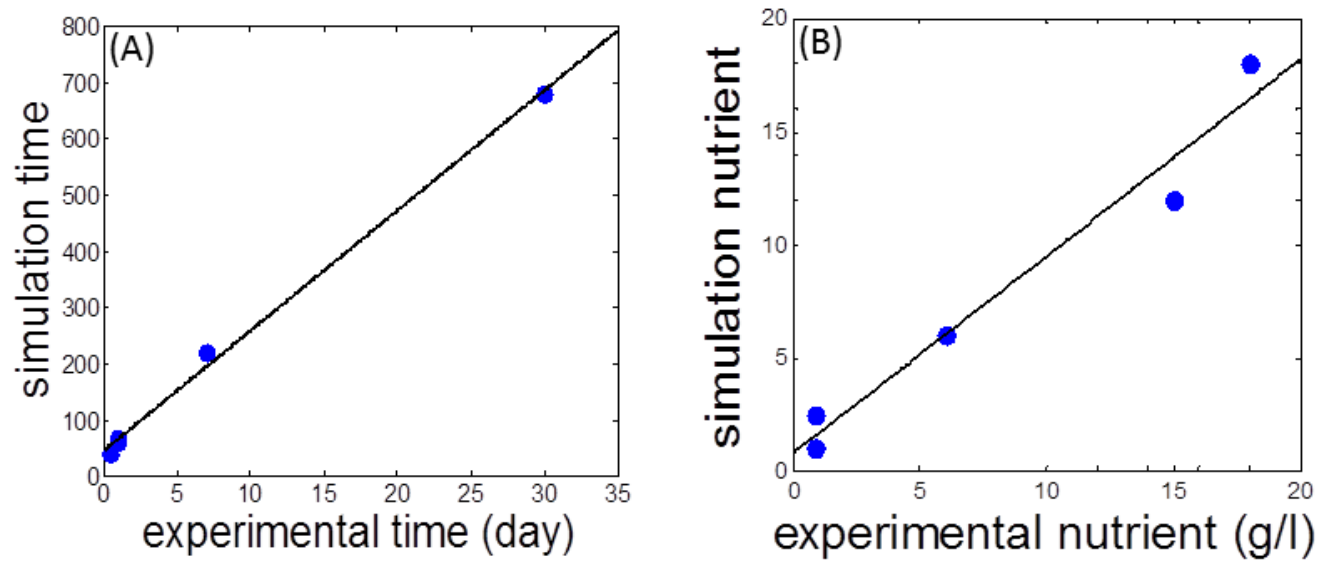

Figure 5 Comparing simulated and experimental values. (A) Colony growth rates. (B) Initial nutrient concentration.

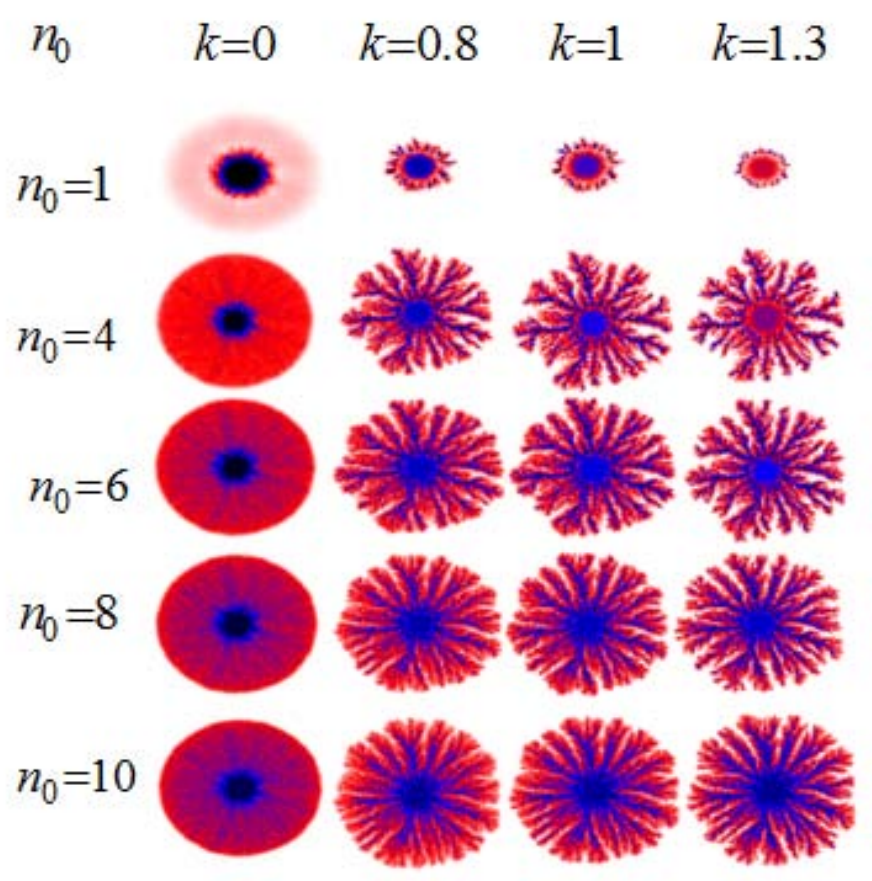

Figure A1 Simulated patterns as function of $k$ and $n_{0}$. 


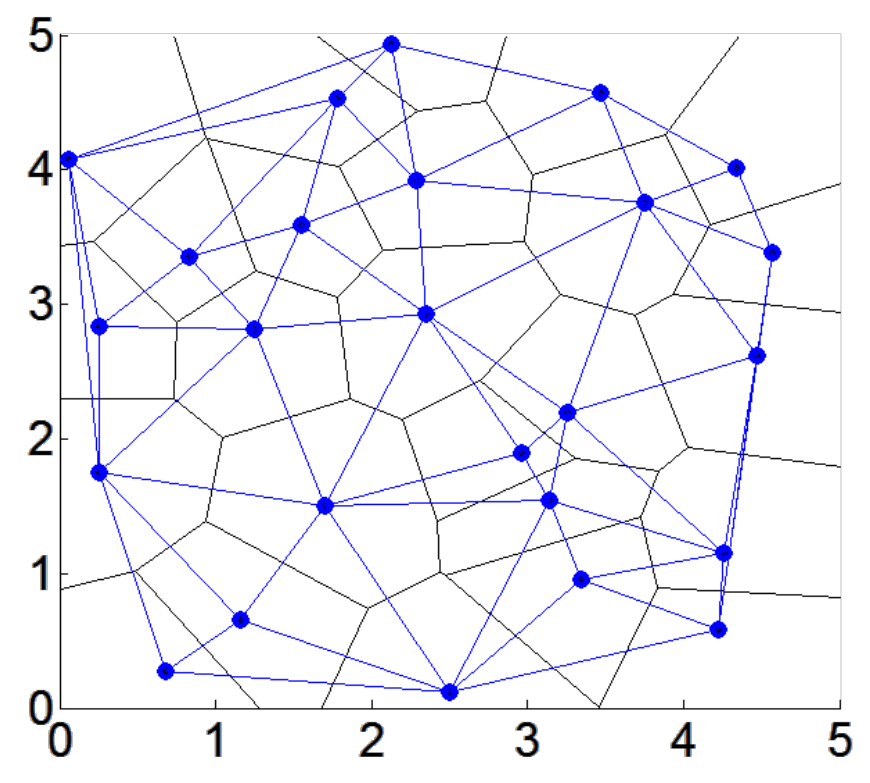

Figure B1 An example of a random computational lattice, generated by the algorithm described previously.

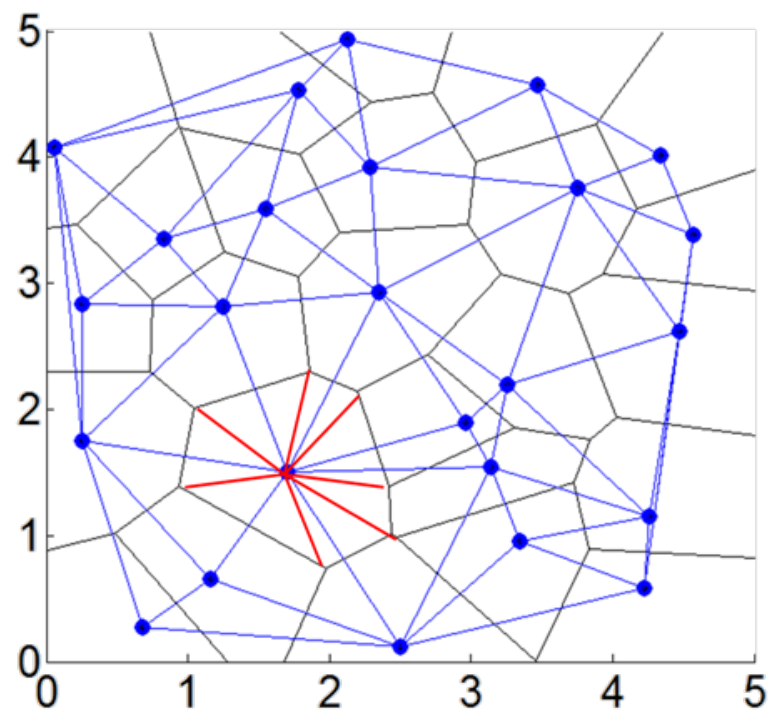

Figure B2 division of a Vornoi cell in to several triangles 


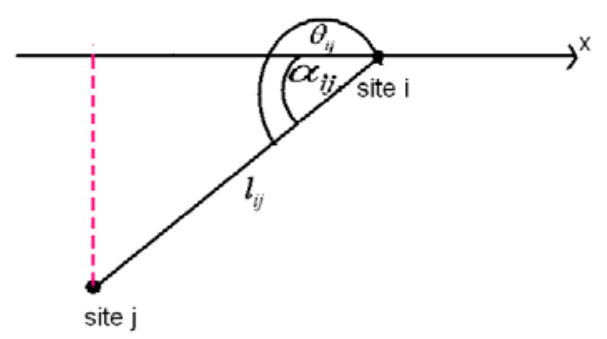

Figure B3 The angle $\alpha_{i j}$ is between the line connecting sites $i$ and $j$ and the $\mathrm{x}$ axis. 
Tables

\begin{tabular}{|l|l|l|l|l|l|}
\hline Pattern & A & B & C & D & E \\
\hline $\begin{array}{l}\text { Agar } \\
\text { concentration }\end{array}$ & $10 \mathrm{gr} / 1$ & $10 \mathrm{gr} / 1$ & $7 \mathrm{gr} / 1$ & $5 \mathrm{gr} / 1$ & $6 \mathrm{gr} / 1$ \\
\hline $\begin{array}{l}\text { Initial nutrient } \\
\text { concentration } \\
\left(n_{0}\right)\end{array}$ & $0.9 \mathrm{gr} / 1$ & $18 \mathrm{gr} / 1$ & $12 \mathrm{gr} / 1$ & $6 \mathrm{gr} / 1$ & $0.9 \mathrm{gr} / 1$ \\
\hline$D_{0}$ & 0.0125 & 0.0125 & $\begin{array}{l}\text { Alternate } \\
\text { between } \\
0.0125 \text { and } \\
0.125\end{array}$ & 0.625 & 0.025 \\
\hline
\end{tabular}

Table I Experimental and simulated parameters for different patterns. 


\begin{tabular}{|c|c|c|c|}
\hline$\underline{\text { Parameter }}$ & $\underline{\text { Simulation value }}$ & $\begin{array}{l}\text { Simulation value in } \\
\text { physical unites }\end{array}$ & $\begin{array}{l}\text { Estimated } \\
\text { experimental value }\end{array}$ \\
\hline Time & Time step & 1.14 [hour] & \\
\hline Final colony diameter & 60 [cells] & $5[\mathrm{~cm}]$ & $5[\mathrm{~cm}]$ \\
\hline $\begin{array}{l}\text { Diffusion coefficient of } \\
\text { chemicals in agar } D_{n}\end{array}$ & $0.25\left[\right.$ length $^{2} /$ time $]$ & $4.6 \cdot 10^{-7}\left[\mathrm{~cm}^{2} / \mathrm{sec}\right]$ & $10^{-7}\left[\mathrm{~cm}^{2} / \mathrm{sec}\right]$ \\
\hline $\begin{array}{l}\text { Reproduction period } \\
T_{r}=1 / \beta\end{array}$ & $0.9-3.33$ [time step] & $61.7-228[\mathrm{~min}]$ & $\begin{array}{l}25[\mathrm{~min}] \text { under } \\
\text { optimal conditions. }\end{array}$ \\
\hline $\begin{array}{l}\text { Consumption period } \\
\text { (time to consume self } \\
\text { weight) } T_{c}=1 / \lambda\end{array}$ & 1.1 [time step] & 1.25 [hour] & $\begin{array}{l}\text { Bacteria requires } \\
\text { about three } \\
\text { time its mass for } \\
\text { reproduction }\end{array}$ \\
\hline Density of bacteria: $b, s$ & $\begin{array}{l}10.61 \\
\text { [weight/surface] }\end{array}$ & $2.8\left[\mathrm{mg} / \mathrm{cm}^{2}\right]$ & $\begin{array}{l}0.12-4.8\left[\mathrm{mg} / \mathrm{cm}^{2}\right. \\
0.12-4.8\left[\mathrm{mg} / \mathrm{cm}^{2}\right]\end{array}$ \\
\hline Density of nutrient, $n$ & $1-18[$ weight/surface $]$ & $0.9-16.2[\mathrm{gr} / 1]$ & $0.8-18[\mathrm{gr} / 1]$ \\
\hline
\end{tabular}

Table II, Correspondence between the simulated and experimental units. The first column lists the parameters appearing in simulations. The second column is the values used in in the numerical solution of equations (1)-(3). The third column is the simulation value, converted to physical units. The last column specifies a range of possible experimental values. 